\title{
Integrated photonic delay-lasers for reservoir computing
}

\section{Van der Sande, Guy, Harkhoe, Krishan, Katumba, Andrew, Bienstman, Peter, Verschaffelt, Guy}

Guy Van der Sande, Krishan Harkhoe, Andrew Katumba, Peter Bienstman, Guy Verschaffelt, "Integrated photonic delay-lasers for reservoir computing," Proc. SPIE 11274, Physics and Simulation of Optoelectronic Devices XXVIII, 112740D (2 March 2020); doi: 10.1117/12.2550576

SPIE. Event: SPIE OPTO, 2020, San Francisco, California, United States 


\title{
Integrated photonic delay-lasers for reservoir computing
}

\author{
Guy Van der Sande ${ }^{\mathrm{a}}$, Krishan Harkhoe ${ }^{\mathrm{a}}$, Andrew Katumba ${ }^{\mathrm{b}}$, Peter Bienstman ${ }^{\mathrm{b}}$, and Guy \\ Verschaffelt ${ }^{\mathrm{a}}$ \\ ${ }^{a}$ Applied Physics Research Group, Vrije Universiteit Brussel, Pleinlaan 2, 1050 Brussels, \\ Belgium \\ ${ }^{b}$ Photonics Research Group, Department of Information Technology, Ghent University-IMEC, \\ Technologiepark Zwijnaarde 126, 9052 Ghent, Belgium
}

\begin{abstract}
Currently, multiple photonic reservoir computing systems show great promise for providing a practical yet powerful hardware substrate for neuromorphic computing. Among those, delay-based systems offer a simple technological route to implement photonic neuromorphic computation. Its operation boils down to a time-multiplexing with the delay length limiting the processing speed. As most optical setups end up to be bulky employing long fiber loops or free-space optics, the processing speeds are ranging from $\mathrm{kSa} / \mathrm{s}$ to tens of $\mathrm{MSa} / \mathrm{s}$. Therefore, we focus on external cavities which are far shorter than what has been realized before in such experiments. We present experimental results of reservoir computing based on a semiconductor laser, operating in a single mode regime around $1550 \mathrm{~nm}$, with a $10.8 \mathrm{~cm}$ delay line. Both are integrated on an active/passive InP photonic chip built on the Jeppix platform. Using 23 virtual nodes spaced 50 ps apart in the integrated delay section, we increase the processing speed to $0.87 \mathrm{GSa} / \mathrm{s}$. The computational performance is benchmarked on a forecasting task applied to chaotic time samples. Competitive performance is observed for injection currents above threshold, with higher pumps having lower prediction errors. The feedback strength can be controlled by electrically pumping integrated amplifiers within the delay section. Nevertheless, we find good performance even when these amplifiers are unpumped. To proof the relevance and necessity of the external cavity on the computational capacity, we have analysed linear and nonlinear memory tasks. We also propose several post-processing methods, which increase the performance without a penalty to speed.
\end{abstract}

Keywords: neuromorphic computing, reservoir computing, delayed feedback, semiconductor laser, photonic integration

\section{INTRODUCTION}

Artificial neural networks (ANN) have played a significant role in the current AI boom, especially with the invention of ImageNet ${ }^{1}$ as catalyst. ANNs may be efficient and versatile during operation, nevertheless they require complex and time-consuming algorithms to train the connection weights in the large network that forms the ANN. When interested in processing tasks and data where the temporal evolution is key, standard feedforward ANNs are not sufficient and one needs to turn to recurrent neural networks (RNNs). The training of RNNs is a nonlinear problem due to feedback loops in the network and is far more involved than the training algorithms of feed-forward networks. Reservoir computing (RC) is paradigm that solves the training issue of RNNs in an efficient way.

$\mathrm{RC}$ offers a framework to exploit the transient dynamics within a RNN for performing useful computation. It has been demonstrated to have state-of-the-art performance for a range of tasks that are notoriously hard to solve by algorithmic approaches, e.g., speech and pattern recognition and nonlinear control. RC simplifies the training procedure for RNNs considerably. Its training procedure only acts on the output layer which consists of a linear combination of network states to generate the desired output signals. The connections of the RNN itself, which is now referred to as reservoir, remain fixed. During training, only the connections from the network

Further author information: (Send correspondence to GVDS)

GVDS: E-mail: guy.van.der.sande@vub.be

Physics and Simulation of Optoelectronic Devices XXVIII, edited by Bernd Witzigmann,

Marek Osiński, Yasuhiko Arakawa, Proc. of SPIE Vol. 11274, 112740D · @ 2020 SPIE

CCC code: $0277-786 \mathrm{X} / 20 / \$ 21 \cdot$ doi: $10.1117 / 12.2550576$

Proc. of SPIE Vol. 11274 112740D-1 
to the output layer are adjusted. Due to this simplification, RC is very suited as framework for neuromorphic computing activities in photonics. Today, multiple photonic RC systems exist that can provide a practical yet powerful hardware substrate for neuromorphic computing. ${ }^{2}$ Some examples include a network of semiconductor optical amplifiers, ${ }^{3,4}$ an integrated passive silicon circuit forming a very complex and random interferometer, with nonlinearity introduced in the readout stage ${ }^{5}$ and a semiconductor laser network based on diffractive coupling. ${ }^{6,7}$ Applications can lie within telecom, ${ }^{8}$ but also classification of ECGs ${ }^{9,10}$ is a possible task among others. All these implementations have one thing in common: they rely on a network of photonic nodes that are spatially distributed and can be measured simultaneously.

However, the reservoir is not required to be a networked structure. In fact, any dynamical system with a high dimensional state space can be considered as reservoir substrate. We consider here specifically a semiconductor laser with delayed feedback as reservoir substrate. The concept of delay-based RC, using only a single nonlinear node with delayed feedback, was introduced some years ago by Appeltant et al. ${ }^{11}$ Delay-based RC offers a simple technological route to implement photonic neuromorphic computation. Its operation boils down to a time-multiplexing with the delay arising from propagation in the external feedback loop, limiting the resulting processing speed. The system is easily scaled by tuning the delay length and only has one single physical node reducing the hardware complexity in photonic systems. The first working prototype was developed in electronics in 2011 by Appeltant et al. ${ }^{11}$ and studied many configurations. ${ }^{12,13}$ Several performant optical systems followed quickly after that. ${ }^{14-16}$ Brunner et al. ${ }^{17}$ employed of-the-shelf telecom equipment to experiment with a singlemode semiconductor laser subjected to optical feedback. The delay time in his experiments was around 80ns, which translates to a few meters of fiber. As most optical setups end up to be bulky employing long fiber loops or free-space optics, the processing speeds are limited in the range of $\mathrm{kSa} / \mathrm{s}$ to tens of $\mathrm{MSa} / \mathrm{s} .{ }^{15,17}$ To increase the processing speed of delay-based reservoir computing using a semiconductor laser with delayed optical feedback, one can integrate the laser and the delay both on the same photonic chip. In this way, by using a waveguide structure with a compact footprint, an external cavity structure can be implemented which is small enough to reach high processing speeds, yet still long enough to have sufficient dimensionality for good computing performance. In the long term, this integrated approach will lead to a robust and low-cost design. Recently, Takano et al. ${ }^{18}$ have presented a photonic integrated circuit consisting of a distributed-feedback semiconductor laser, a semiconductor optical amplifier (SOA), a phase modulator, a short passive waveguide, and an external mirror for optical feedback. The external cavity length in this system reached $10.6 \mathrm{~mm}$, corresponding to a round-trip delay time of 254ps. However, only six virtual nodes could be stored within the delay line with node-spacings of $40 \mathrm{ps}$, not enough for good computational performance. This necessitated the authors to use masks with duration of multiple delay times, which slows down the computation speed. Several other types of semiconductor laser have been considered such as semiconductor ring lasers using the two available directional modes ${ }^{19-26}$ and vertical-cavity surface-emitting employing the two polarization modes ${ }^{27-30}$ and multi-mode lasers employing longitudinal modes. ${ }^{31}$ In this paper, we will only focus on single-mode Fabry-Péro type quantum-well semiconductor lasers.

The information processing performance of a semiconductor laser-based RC system is related to its dynamical behaviour both in the absence of external input and in the presence of said input. After the very first experiment by Brunner et al., ${ }^{17}$ other works have focused on understanding the fundamental properties of semiconductor laser-based RC for non-linear prediction tasks. In Ref. 32, it has been shown that the conditions to achieve good predictive performance are given by the injection locking, consistency, and memory properties of the system. More specifically, Bueno et al. found that the lowest prediction error for a non-linear prediction task occurs at the injection locking boundary. Note that in this work the laser was operating below or close to the solitary lasing threshold. Consistency, the ability of a system to have a similar response for the similar input signals, is widely regarded as key for good reservoir computing performance. ${ }^{32,33}$

Our goal is to show that a delay-based reservoir computer can be built using an indium-phosphide PIC, that combines active and passive elements and is built on the JePPIX platform. ${ }^{34}$ The PIC integrates a semiconductor laser with an external cavity of $5.4 \mathrm{~cm}$, which corresponds to a round trip time of $1170 \mathrm{ps}$. This allows for 23 nodes and a processing speed of $0.87 \mathrm{GSa} / \mathrm{s}$. The longer waveguide based external cavity will also have more loss associated to it. Therefore, we will address in this work the question if amplification in the external cavity is needed or not. Contrary to other works, ${ }^{17,18,32}$ the semiconductor laser itself will be driven far above solitary 


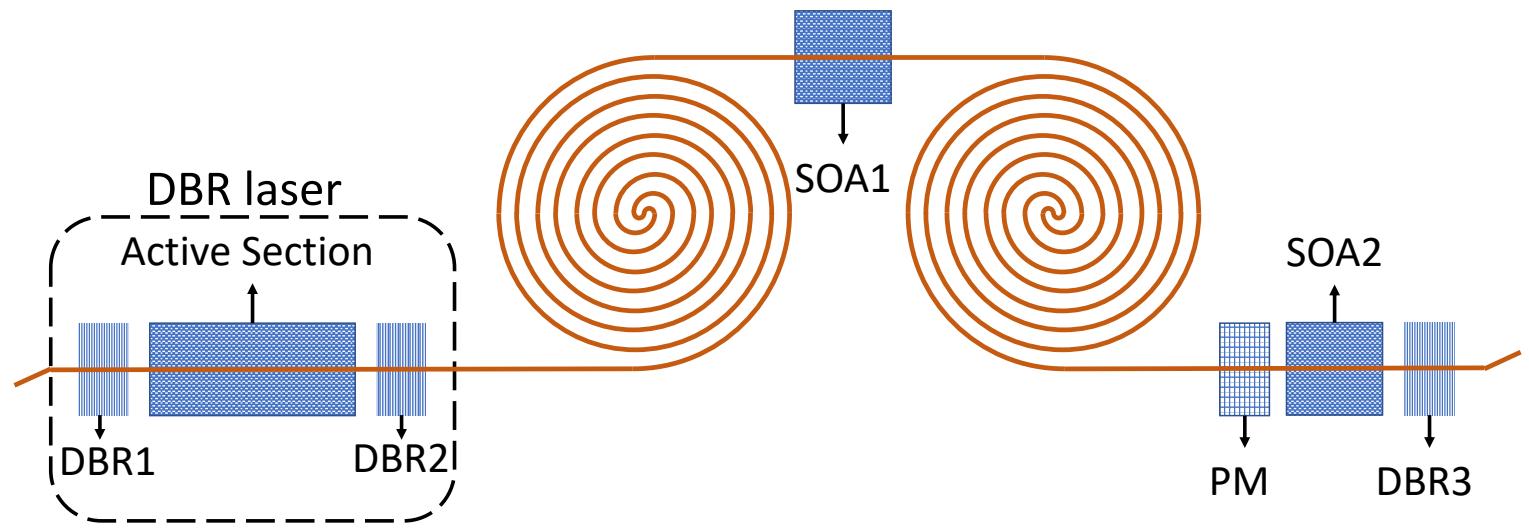

Figure 1. Schematic depiction of our InP-based photonic integrated circuit (PIC). The PIC consists of a laser structure followed by a delay line of $5.4 \mathrm{~cm}$. DBR: Distributed Bragg Reflector, SOA: Semiconductor Optical Amplifier, PM: Phase Modulator.

lasing threshold to benefit from a better signal to noise ratio in the read-out, as well as faster internal dynamics. Finally, we will introduce post-processing schemes that do not penalize computational speed

\section{EXPERIMENTAL SETUP}

A schematic of our integrated device is shown in Fig. 1. It consists of a distributed Bragg reflector (DBR) laser structure and two spiral waveguides comprising the delay line. Two semiconductor optical amplifiers (SOA) are placed along the delay line to tune the feedback strength. A phase modulator is available to tune the feedback phase. We have not used this phase modulator due to setup complexity. Nevertheless, the phase is expected to play an important role. ${ }^{35}$ At the end of the delay line a DBR element completes the feedback loop by reflection. This on-chip feedback loop has a round-trip time of $\tau=1170 \mathrm{ps}$.

The device covers the whole $6 \mathrm{~mm}$ width of the chip and has one optical input/output port on each side. The ports are angled with respect to the chip edge to minimize reflection. We employed lensed fibers to send optical signals in/out of these ports and a total of five electrical DC probes to operate the device. The first probe $\left(I_{D B R 1}\right)$ was placed on the left DBR of the laser structure, in order to tune the spectral output of the laser. The second probe $\left(I_{L}\right)$ acted to supply the pump current to the laser. The following two probes $\left(I_{S O A 1}, I_{S O A 2}\right)$ supplied current to the SOAs along the feedback line and the last probe $\left(I_{D B R 3}\right)$ tuned the reflection spectrum of the DBR at the end of the feedback line. The active and SOA sections could be pumped up to a current of $40 \mathrm{~mA}$, whereas the tuning currents of the DBRs could only be driven up to $10 \mathrm{~mA}$.

The DBR laser has a threshold current of $15 \mathrm{~mA}$. The free running lasing wavelength is centered at $1546.91 \mathrm{~nm}$. In our setup, the on-chip laser can lock on the injection at the free running lasing wavelength or one of the sidemodes, depending on the injected wavelength. It turned out that the RC performance is best when the injected field's wavelength is close to a side mode. Targeting the side mode allows for a higher injected power as the reflection of DBR1 is lower at the wavelength of the side-mode. Furthermore, DBR2 has a higher transmission for side modes than for the free running lasing wavelength. Injection locking on the side-mode is achieved at a wavelength of $1549.60 \mathrm{~nm}$ and the following DC probes configuration: $I_{D B R 1}=8.28 \mathrm{~mA}, I_{D B R 3}=1 \mathrm{~mA}$, and $I_{L}=I_{S O A 1}=I_{S O A 2}=40 \mathrm{~mA}$. The on-chip spectral parameters are not changed hereafter, meaning that the current supply to the two DBRs is not changed throughout the paper. A similar chip was recently used for random number generation. ${ }^{36}$

To test the RC performance of the laser integrated with a feedback loop, the setup shown in Fig. 2 is used. We use a wavelength tuneable $\mathrm{CW}$ laser to create the optical injection signal. The wavelength of this laser is set close to $1549.6 \mathrm{~nm}$, but we still allow for a small detuning between the injection wavelength and the wavelength of the targeted side-mode of the laser. The $\mathrm{CW}$ light beam of the tunable laser is modulated using a 40GHz MachZehnder modulator (iXblue MX-LN-40). This modulator is driven electrically by a $25 \mathrm{GHz}$ Arbitrary Waveform Generator (Keysight M8195A) set at a sample speed of $60 \mathrm{GSa} / \mathrm{s}$. 


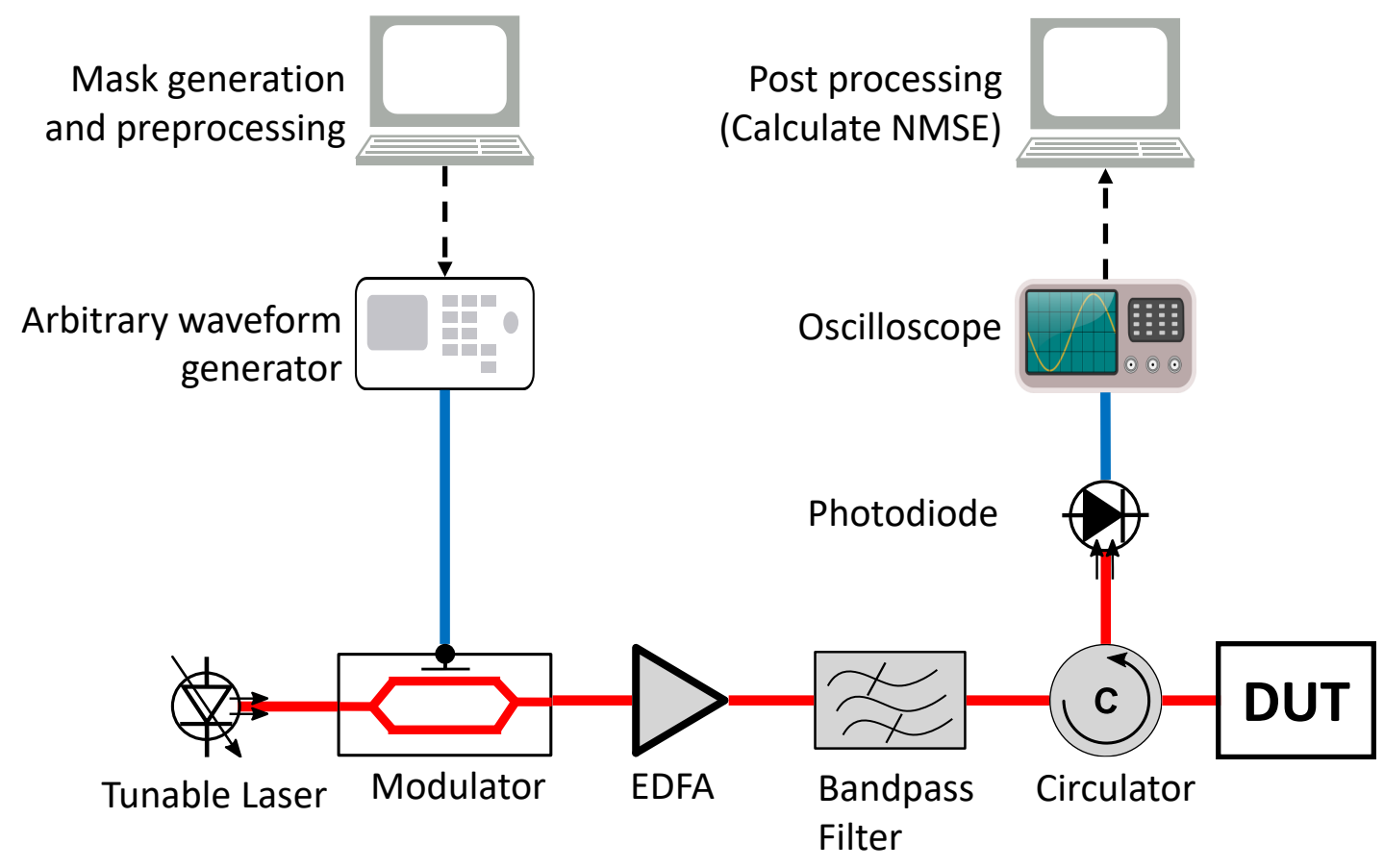

Figure 2. Schematic depiction of the setup used to measure the performance of our integrated delay-based reservoir computer. The device under test (DUT) is the PIC shown in Fig. 1

We employ the time-multiplexing scheme ${ }^{37}$ as introduced in 11 , where the duration of one data sample matches the 1170 ps delay time. Note that there have been numerical and experimental studies, where the duration of a data sample does not match the delay time. ${ }^{15,38}$ We, however, do not target this working regime. A similar time-multiplexing scheme can also be used for coherent ising machines. ${ }^{39}$

Any input data sample $u_{i}$, in our case originating from a discrete timeseries, is held constant for the duration of one delay time $\tau$. We then multiply this piecewise constant stream $U(t)$ with a piecewise constant mask $M(t)$ (that is periodic with a period of $\tau$ ) to obtain the masked input stream $J(t)$. The piecewise constant levels of stream $J(t)$ define the position of the virtual nodes equally spread over the delay line. It has been shown numerically ${ }^{40}$ that the node separation, when using a semiconductor laser with delayed feedback, can be as short as a few tens of ps. As the sample rate of the AWG is set to $60 \mathrm{GSa} / \mathrm{s}$, we use three AWG samples to define one mask node, leading to a mask node separation of $\theta_{M}=50 \mathrm{ps}$ such that 23 nodes fit within one round-trip in the delay loop. We thus generate a random mask with $N_{M}=23$ mask nodes with three possible values $[0,0.5,1]$. In our case the length of the mask is 20 ps shorter than the delay time, which is hard to match in practice. We believe this desynchronization will not adversely affect the performance of the RC scheme, since the mismatch is smaller than the node separation and we can accurately split the reservoir output in the readout layer. ${ }^{41}$

The modulated optical signal is next amplified in Fig. 2 using an Erbium doped fiber amplifier (Keopsys CEFA-C-BO-HP-B203). The broadband spontaneous emission noise, added to the optical signal by the amplifier, is removed by sending the light beam through an optical bandpass filter, that is centered around the injection signal's wavelength. The filtered signal is then fed into the laser using a circulator connected to a lensed fiber. The response of the laser is collected at the third port of the circulator and measured using an opto-electronic detector connected to a $63 \mathrm{GHz}$ real-time oscilloscope. The sampling rate of the oscilloscope was set to $40 \mathrm{GSa} / \mathrm{s}$. This means that each mask node, with a duration of $50 \mathrm{ps}$, has 2 corresponding samples in the read-out signal. 


\section{RESULTS}

We have studied the performance of a delay-based reservoir computer, which is designed on a photonic integrated chip. The integrated approach leads to a compact design as well as high computation speeds. We have studied the performance through the Santa-Fe timeseries benchmarking task.

With a conventional reservoir computing scheme, where the mask-imposed nodes coincide with the virtual nodes, we get a performance (best $N M S E=0.135$ ) which is slightly worse than those found in other works (NMSE around 0.1). However, we are working in different regimes. While previous works, such as, ${ }^{17,18,32,40}$ operate in sub- or near threshold regimes, we operate our laser at pump currents well above the threshold current. We achieve a significant speed up compared to others, ${ }^{14,17}$ who achieved speeds in the order of $\mathrm{kSa} / \mathrm{s}$ and MSa/s respectively. The computation speed of our setup is $0.87 \mathrm{GSa} / \mathrm{s}$, which is comparable to what Takano et al. achieved with additional pre- and post-processing steps.

We were able to improve the performance of the reservoir computer by using different post-processing routines. The first routine is using both readout samples within one mask-imposed node to form the output layer, unlike the conventional routine where we utilize one sample per mask-imposed node. The availability of extra states in the output layer, causes the reservoir computer to perform better. The extra states are not redundant in comparison with the rest, but rather enhance the state space. Since the mask-imposed node has a slightly longer duration than the timescale of the laser, we get two different state values from the transient response on the input. The best performance we achieved here is $N M S E=0.062$.

The second post-processing routines takes the reservoir output for a duration of two delay times. This way we have a richer state space to perform the task and furthermore have access to a longer temporal memory inside this state space, since the last two input data points are present in the two delay times. This post-processing routine has consistently been the best performing out of the three and reaches an NMSE as low as 0.049.

We have seen that the best performance for Santa Fe timeseries prediction was found when we the injected signal's wavelength was close to a side-mode, with zero detuning between the injected wavelength and side-mode. We also observed that delay-based RC using semiconductor lasers can achieve very good performances at pump currents well above threshold, where most studies have focused on near-threshold operation. Lastly, we studied the memory capacity of our RC setup as the feedback in the setup is increased and we see a clear increase. Even when the SOAs in the delay line are turned off, we get a linear memory capacity around 8, which suggests that there is enough feedback already in the system without extra amplification. Possibly the perfomance can be improved further if this system were to be cascaded. ${ }^{42}$

\section{ACKNOWLEDGEMENTS}

We would like to acknowledge funding from the Research Foundation Flanders (FWO) through grants G028618N, G024715N, G029519N and G006020N, from the EU Horizon 2020 PHRESCO Grant (688579) and the EU Horizon 2020 Fun-COMP Grant (780848). Finally, we would like to thank BELSPO for funding through IAP P7-35 program Photonics@be, the Hercules Foundation and the Research Council of the VUB.

\section{REFERENCES}

[1] Krizhevsky, A., Sutskever, I., and Hinton, G. E., "Imagenet classification with deep convolutional neural networks," in [Advances in neural information processing systems], 1097-1105 (2012).

[2] Van der Sande, G., Brunner, D., and Soriano, M. C., "Advances in photonic reservoir computing," Nanophotonics 6, 561-576 (2017).

[3] Vandoorne, K., Dierckx, W., Schrauwen, B., Verstraeten, D., Baets, R., Bienstman, P., and Van Campenhout, J., "Toward optical signal processing using photonic reservoir computing," Opt. Express 16, 11182$11192(2008)$.

[4] Vandoorne, K., Dambre, J., Verstraeten, D., Schrauwen, B., and Bienstman, P., "Parallel reservoir computing using optical amplifiers," IEEE Trans. Neural Netw. 22, 1469-1481 (2011).

[5] Vandoorne, K., Mechet, P., Van Vaerenbergh, T., Fiers, M., Morthier, G., Verstraeten, D., Schrauwen, B., Dambre, J., and Bienstman, P., "Experimental demonstration of reservoir computing on a silicon photonics chip," Nat. Commun. 5 (2014). 
[6] Brunner, D. and Fischer, I., "Reconfigurable semiconductor laser networks based on diffractive coupling," Opt. Lett. 40, 3854-3857 (2015).

[7] Maktoobi, S., Froehly, L., Andreoli, L., Porte, X., Jacquot, M., Larger, L., and Brunner, D., "Diffractive Coupling For Photonic Networks: How Big Can We Go?," IEEE J. Sel. Top. Quantum Electron. 26 (2020).

[8] Argyris, A., Cantero, J., Galletero, M., Pereda, E., Mirasso, C. R., Fischer, I., and Soriano, M. C., "Comparison of Photonic Reservoir Computing Systems for Fiber Transmission Equalization," IEEE J. Sel. Top. Quantum Electron. 26 (2020).

[9] Alfaras, M., Soriano, M. C., and Ortin, S., "A Fast Machine Learning Model for ECG-Based Heartbeat Classification and Arrhythmia Detection," Front. Phys. 7 ( 2019).

[10] Ortin, S., Soriano, M. C., Alfaras, M., and Mirasso, C. R., "Automated real-time method for ventricular heartbeat classification," Comput. Methods Programs Biomed. 169, 1-8 (2019).

[11] Appeltant, L., Soriano, M. C., Van der Sande, G., Danckaert, J., Massar, S., Dambre, J., Schrauwen, B., Mirasso, C. R., and Fischer, I., "Information processing using a single dynamical node as complex system," Nat. Commun. 2, 468 (2011).

[12] Soriano, M. C., Ortin, S., Keuninckx, L., Appeltant, L., Danckaert, J., Pesquera, L., and Van der Sande, G., "Delay-Based Reservoir Computing: Noise Effects in a Combined Analog and Digital Implementation," IEEE Trans. Neural Netw. Learn. Syst. 26, 388-393 (2015).

[13] Penkovsky, B., Porte, X., Jacquot, M., Larger, L., and Brunner, D., "Coupled Nonlinear Delay Systems as Deep Convolutional Neural Networks," Phys. Rev. Lett. 123 (2019).

[14] Paquot, Y., Dambre, J., Schrauwen, B., Haelterman, M., and Massar, S., "Reservoir computing: a photonic neural network for information processing," in [SPIE Photonics Europe], 77280B-77280B, International Society for Optics and Photonics (2010).

[15] Paquot, Y., Duport, F., Smerieri, A., Dambre, J., Schrauwen, B., Haelterman, M., and Massar, S., "Optoelectronic reservoir computing," Sci. Rep. 2 (2012).

[16] Pauwels, J., Verschaffelt, G., Massar, S., and Van der Sande, G., "Distributed Kerr Non-linearity in a Coherent All-Optical Fiber-Ring Reservoir Computer," Front. Phys. 7, 17597 (2019).

[17] Brunner, D., Soriano, M. C., Mirasso, C. R., and Fischer, I., "Parallel photonic information processing at gigabyte per second data rates using transient states," Nat. Commun. 4, 1364 (2013).

[18] Takano, K., Sugano, C., Inubushi, M., Yoshimura, K., Sunada, S., Kanno, K., and Uchida, A., "Compact reservoir computing with a photonic integrated circuit," Opt. Express 26, 29424-29439 (2018).

[19] Nguimdo, R. M., Verschaffelt, G., Danckaert, J., and Van der Sande, G., "Simultaneous computation of two independent tasks using reservoir computing based on a single photonic nonlinear node with optical feedback," IEEE Trans. Neural Netw. Learn. Syst. 26, 3301-3307 (2015).

[20] Mashal, L., Van der Sande, G., Gelens, L., Danckaert, J., and Verschaffelt, G., "Square-wave oscillations in semiconductor ring lasers with delayed optical feedback," Opt. Express 20, 22503-22516 (2012).

[21] Ermakov, I. V., Van der Sande, G., and Danckaert, J., "Semiconductor ring laser subject to delayed optical feedback: Bifurcations and stability," Commun. Nonlinear Sci. Numer. Simul. 17, 4767-4779 (2012).

[22] Coomans, W., Gelens, L., Van der Sande, G., Mezosi, G., Sorel, M., Danckaert, J., and Verschaffelt, G., "Semiconductor ring lasers coupled by a single waveguide," Appl. Phys. Lett. 100, 251114 (2012).

[23] Gelens, L., Mashal, L., Beri, S., Coomans, W., Van der Sande, G., Danckaert, J., and Verschaffelt, G., "Excitability in semiconductor microring lasers: Experimental and theoretical pulse characterization," Phys. Rev. A 82, 063841 (2010).

[24] Gelens, L., Beri, S., Van der Sande, G., Verschaffelt, G., and Danckaert, J., "Multistable and excitable behavior in semiconductor ring lasers with broken Z(2)-symmetry," Eur. Phys. J. D 58, 197-207 (2010).

[25] Gelens, L., Van der Sande, G., Beri, S., and Danckaert, J., "Phase-space approach to directional switching in semiconductor ring lasers," Phys. Rev. E 79, 016213 (2009).

[26] Gelens, L., Beri, S., Van der Sande, G., Danckaert, J., Calabretta, N., Dorren, H. J. S., Notzel, R., Bente, E. A. J. M., and Smit, M. K., "Optical injection in semiconductor ring lasers: backfire dynamics," Opt. Express 16, 10968-10974 (2008).

[27] Vatin, J., Rontani, D., and Sciamanna, M., "Enhanced performance of a reservoir computer using polarization dynamics in vcsels," Opt. Lett. 43, 4497-4500 (2018). 
[28] Van der Sande, G., Danckaert, J., Veretennicoff, I., and Erneux, T., "Rate equations for vertical-cavity surface-emitting lasers," Phys. Rev. A 67, 013809 (2003).

[29] Van der Sande, G., Verschaffelt, G., Danckaert, J., and Mirasso, C., "Ghost stochastic resonance in verticalcavity surface-emitting lasers: Experiment and theory," Phys. Rev. E 72, 016113 (2005).

[30] Van der Sande, G., Peeters, M., Veretennicoff, I., Danckaert, J., Verschaffelt, G., and Balle, S., "The effects of stress, temperature, and spin flips on polarization switching in vertical-cavity surface-emitting lasers," IEEE J. Quantum Electron. 42, 898-906 (2006).

[31] Harkhoe, K. and Van der Sande, G., "Delay-Based Reservoir Computing Using Multimode Semiconductor Lasers: Exploiting the Rich Carrier Dynamics," IEEE J. Sel. Top. Quantum Electron. 25, 1502909 (2019).

[32] Bueno, J., Brunner, D., Soriano, M. C., and Fischer, I., "Conditions for reservoir computing performance using semiconductor lasers with delayed optical feedback," Opt. Express 25, 2401-2412 (2017).

[33] Kuriki, Y., Nakayama, J., Takano, K., and Uchida, A., "Impact of input mask signals on delay-based photonic reservoir computing with semiconductor lasers," Opt. Express 26, 5777-5788 (2018).

[34] Leijtens, X., "Jeppix: the platform for indium phosphide-based photonics," IET optoelectronics 5, 202-206 (2011).

[35] Nguimdo, R. M., Verschaffelt, G., Danckaert, J., and Van der Sande, G., "Reducing the phase sensitivity of laser-based optical reservoir computing systems," Opt. Express 24, 1238-1252 (2016).

[36] Verschaffelt, G., Khoder, M., and Van der Sande, G., "Random number generator based on an integrated laser with on-chip optical feedback," Chaos 27, 114310 (2017).

[37] Brunner, D., Penkovsky, B., Marquez, B. A., Jacquot, M., Fischer, I., and Larger, L., "Tutorial: Photonic neural networks in delay systems," J. Appl. Phys. 124 (2018).

[38] Duport, F., Smerieri, A., Akrout, A., Haelterman, M., and Massar, S., "Fully analogue photonic reservoir computer," Sci. Rep. 6, 22381 (2016).

[39] Bohm, F., Verschaffelt, G., and Van der Sande, G., "A poor man's coherent Ising machine based on optoelectronic feedback systems for solving optimization problems," Nat. Commun. 10, 3538 (2019).

[40] Nguimdo, R. M., Verschaffelt, G., Danckaert, J., and Van der Sande, G., "Fast photonic information processing using semiconductor lasers with delayed optical feedback: role of phase dynamics," Opt. Express 22, 8672-8686 (2014).

[41] Harkhoe, K. and Van der Sande, G., "Task-independent computational abilities of semiconductor lasers with delayed optical feedback for reservoir computing," Photonics 6, 124 (2019).

[42] Keuninckx, L., Danckaert, J., and Van der Sande, G., "Real-time Audio Processing with a Cascade of Discrete-Time Delay Line-Based Reservoir Computers," Cogn. Comput. 9, 315-326 (2017). 